\title{
Parameter estimation for subsoil modelling via seismic refraction
}

\author{
Bibiana Boada, Universidad EAFIT, Olga Quintero, Universidad EAFIT, Juan Paniagua, Instituto Tecnológico Metropolitano
}

Copyright 2019, SBGf - Sociedade Brasileira de Geofísica

This paper was prepared for presentation during the $16^{\text {th }}$ International Congress of the Brazilian Geophysical Society held in Rio de Janeiro, Brazil, 19-22 August 2019.

Contents of this paper were reviewed by the Technical Committee of the $16^{\text {th }}$ International Congress of the Brazilian Geophysical Society and do not necessarily represent any position of the SBGf, its officers or members. Electronic reproduction or storage of any part of this paper for commercial purposes without the written consent of the Brazilian Geophysical Society is prohibited.

\section{Abstract}

For mining exploration in geologically complex zones, there are several techniques, among them the seismic refraction, which studies the propagation of seismic waves produced artificially, establishing its relationship with the geological configuration of the subsoil. One of these techniques is the Generalized Reciprocal Method (GRM) which has been proposed for reconstruction of subsurface structures with lateral variations of velocity. This method depends mainly on a single parameter called the optimum $X Y$ distance. This optimum $X Y$ value presents uncertainties when this is determined. In this work, we proposed a robust mathematical way to find the optimal $X Y$ value in order to reduce uncertainties in such a way that a better delineation of the subsurface structures is achieved

\section{Introduction}

The seismic refraction method is a non-destructive geophysical method, used for interpretation of properties of the soil, and the detection of the depth of the rock, by analyzing variations in the seismic velocities (FRANCO, 2016). It is a handy method for investigation of the geological structure, properties of the land, problems of foundations, as well as the study of alluvial layers, landslides, gravel pits, and sand pits, among others (CHILUSA \& NARANJO, 2014).

The interpretation techniques for data obtained with seismic refraction are very varied. For the simple case where the refractor is flat, and the velocity in layers are increased with depth, corrected refraction times can be converted to depth using time-distance curves. In practice, the geology is generally more complicated than this simple case, but a model of reasonable depth can often be obtained using this simplistic approach.

Time-distance curves obtained from a seismic refraction study contain information of velocity and structure of the refractor and, to some extent, on upper layers. It is essential that the analysis of a specific refractor uses only arrivals associated with that refractor; this may not be the case if the wrong displacement range is used because arrivals may include those of a deeper or shallower layer.

One of these interpretation techniques is the generalized reciprocal method (GRM). The generalized reciprocal method (GRM) requires arrival times of direct and inverse refraction. With these arrival times in some occasions, approximations of the velocity of layers can be made, as it is the case of intercept time method. When approximations cannot be made, or the velocity of all layers above the refractor are not known, an estimation of the refractor velocity can be obtained.

The travel times in two geophones, separated by a variable distance $X Y$, are used in the analysis of the refractor velocity and in the calculation of time and depth.

At the optimal $X Y$ spacing, the rising ray segments that travel to each geophone emerge near the same point in the refractor. This makes the velocity analysis of the refractor the simplest and depths and times that it shows contain the greatest amount of detail.

GRM that has a $X Y$ value equal to zero is especially prone to produce numerous fictitious changes of the refractor velocity, as well as to produce a coarse smoothing of the topography of the irregular refractor.

With non-optimal $X Y$ values, the velocity analysis function can indicate refractor velocity changes that vary with the $X Y$ separation, both in magnitude and in sign. These fictitious velocity changes generally occur with an irregular refractor topography. The $X Y$ value, for which the velocity analysis function is the simplest, corresponds to the optimal value. The non-optimal $X Y$ value also results in the smoothing of the time-depth for the topography of the irregular refractor. The $X Y$ value for which the time-depths show the most detail corresponds to the optimum value (PALMER, 1980).

To determine the optimal $X Y$ value, Palmer (2001) proposed two different approaches but these are criticized by many authors (LEUNG, 1995, 2003, SJORGEN, 2000, WHITELEY, 2006)

We propose a mathematical method that allows to reduce the uncertainty associated with the location of sensors (Value $X Y$ ) that are used in the reconstruction of the subsurface structures by means of seismic refraction techniques, specifically the Generalized Reciprocal Method (GRM).

\section{Method}

\section{Generalized Reciprocal Method (GRM)}

The generalized reciprocal method (GRM) is a technique for delineating undulating refractors, at any depth, from inline seismic refraction data consisting of forward and reverse traveltimes.

The arrival times at two geophone positions, separated by a variable distance $X Y$, are used in refractor velocity analysis and time-depth calculations. At the optimum $X Y$ value, the rays to each geophone emerge from near the same point on the refractor. The GRM can define layers 
with varying thicknesses and seismic velocities (PALMER, 1980, 2001).

To carry out this, it is necessary to calculate the $X Y$ value, which is obtained using the velocity analysis function and the generalized time-depth values.

\section{Velocity analysis function}

The velocity analysis function, $t_{v}$, is given by

$$
t_{v}=\frac{1}{2}\left(t_{A Y}-t_{B X}+t_{A B}\right)
$$

where $t_{A Y}$ is the time travel from source $\mathrm{A}$ to receiver $\mathrm{B}$, $t_{B X}$ is the time travel from source $\mathrm{B}$ to receiver $\mathrm{X}$, and $t_{A B}$ is the time travel from source $A$ to source $B$ or the reciprocal time (Figure 1).

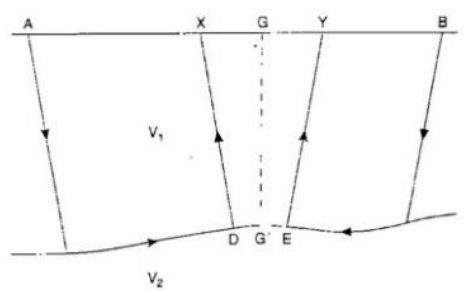

Figure 1. Geometry of the trajectory for the generalized reciprocal method

It is considered that the velocity analysis function is applied at the location $\mathrm{G}$, which is midway between $\mathrm{X}$ and $\mathrm{Y}$.

The optimal value of the distance between $X$ and $Y(X Y)$ occurs when both on the surface measure the time of the two emergent rays corresponding to the same refractor point, that is, D, G ', E coincide.

\section{Time-depth function}

The generalized time-depth function, $t_{G}$, is given by

$$
t_{G}=\frac{1}{2}\left[t_{A Y}+t_{B X}-\left(t_{A B}+\frac{X Y}{V_{n}^{\prime}}\right)\right]
$$

where $V_{n}^{\prime}$ is the apparent velocity of the refractor for the $n t h$ layer, which is defined as the slope of a line fitted to the $t_{v}$ values for the optimum $X Y$ (that for which the forward and the reverse rays emerge from nearly a common point on the refractor). For practical reasons, the values of the generalized time-depth function are often calculated for a range of $X Y$ values at the same time as the velocity analysis function.

\section{Distance between sensors. $X Y$ Value}

Accurate determination of the $X Y$ value is often difficult, especially if surface characteristics in the refractor are small. Under these conditions, the $X Y$ value can be calculated if enough information is available about refractive layers

A general way to determine the $X Y$ value for multiple layers is given by

$$
X Y=2 \sum_{j=1}^{n-1} Z_{j G} \tan \left(\sin ^{-1} \frac{V_{j}}{V_{n}}\right)
$$

where $V_{n}$ is the velocity of the refractor, $Z_{j G}$ is the thickness of the layer, $V_{j}$ is the velocity of the layer, $j$ is the index of the layer, and $n$ is the number of layers.

The time-depth profile $\left(t_{G}\right)$ updated with the estimated $X Y$ value and the refractor velocity as defined in Eq. 3 , is now converted to depth.

An alternative method to convert the generalized timedepth function to depth is used the concept of average velocity (PALMER, 2001). The use of the concept of average velocity requires that the optimal value of $X Y$ will be calculated correctly.

\section{Optimal value of $X Y$}

The determination of optimal $X Y$ values is probably the most difficult and most important aspect of the GRM. There are two different approaches: the first approach is the direct calculation from seismic velocities and thicknesses using Eq. 3 and the second approach is the inspection of time travel data, amplitudes of the seismic trails or the analysis of the refractor velocity and the time and depth functions (PALMER, 2001).

The existence of these two basic approaches to computation and observation of optimal $X Y$ values makes GRM a unique and extremely powerful interpretation method. If the depth is consistent with the travel time data, $X Y$ values calculated and observed should be similar. If these values do not match, it is indicated that there are layers not detected.

For each value $X Y$ you can obtain a different velocity function and depending on the choice you will find a velocity of the medium. A bad choice of $X Y$ makes the velocity of the medium look highly modified.

To determine the optimal $X Y$, we propose to approximate the resulting points in each velocity function to a polynomial of degree one to then measure the error and thus determine which curve is more linear from the group of resulting curves for each possible $X Y$.

With this you get the velocity of the medium using Eq. 1 and the optimal value $X Y$ optimal used to find the depth of the layer. The problem may then be the correct choice of a function to approximate values of $X Y$ in a family.

\section{Uncertainties}

The model that describes the phenomenon is completely deterministic, while the nature of the problem contains very high uncertainties that make it not completely well represented.

Consequently, the propagation of mechanical waves in the environment can be represented with a static model of rays as used in GRM, but this model must contain a component that summarizes the source of uncertainty.

In general, optimal filtering techniques are used to estimate states of a dynamic system whose inputs and outputs are observed by measurements disturbed by noise. It is understood by "states of a system" to the minimum necessary information in a time zero that jointly to the value of entrances defined in all time from $t \geq t_{0}$; allows you to determine the behavior of the system for any $t \geq t_{0}$. 
The measurements are generally uncertain, so we speak of "measurement noise" even if we know the true states of the system, the measurements are not a deterministic function of these states but have a random component.

In this context, the evolution of the states is modeled by a dynamic system disturbed by a stochastic process (noise of the states) by means of a stochastic differential equation. The noise or disturbance of states is incorporated into the model to represent uncertainties of the system, which may be of a random nature, as well as signals or dynamics not considered in the model.

Therefore, we incorporate noise to the position of geophones or to recorded arrival times to find an optimal $\mathrm{XY}$, each time the noise is applied to the phenomenon described

\section{Parameters}

The direct, refracted and reflected waves can be modeled with an uncertainty $\zeta$

$$
t=f_{i}\left(\bar{X}, \theta_{n}\right)+\zeta
$$

where $\bar{X}=\left(x_{1}, x_{2}, \ldots, x_{j}\right)$ is the vector of distances; $\theta_{n}=$ $(\bar{V}, \bar{z})$ are parameters; $\bar{V}=\left(V_{1}, V_{2}, \ldots, V_{n}\right)$ is the velocity vector; $\bar{z}=\left(z_{1}, z_{2}, \ldots, z_{n-1}\right)$ is the vector of depth.

The equation for the case of the refracted wave for $n$ layers is given by

$$
\begin{array}{r}
t_{n-1}=\frac{\Delta x_{i}}{V_{n}}+\frac{2}{V_{n}} \sum_{i=1}^{n-1} h_{i} \frac{\left(V_{n}^{2}-V_{i}^{2}\right)^{1 / 2}}{V_{i}} \\
=f_{3}\left(X, \theta_{n}\right)+\zeta
\end{array}
$$

where $\zeta$ is a random variable that can be of uniform, Gaussian or colored distribution and, therefore, generates a family of optimal curves which can be treated with functional data.

\section{Optimum $X Y$ value}

We obtain a set of optimal curves from which the functional median is searched using functional data tools such as those incorporated by Fraiman \& Muniz (2001) where their aim was to find the most central curve of a group of curves, resulting in a robust optimal $X Y$.

The value $X Y$ is very important in the GRM, in this case, the velocity function (Eq. 1) is calculated for all possible values of $X Y$ which varies from zero to half of the distance of disturbances $A$ and $B$ (Figure 2).

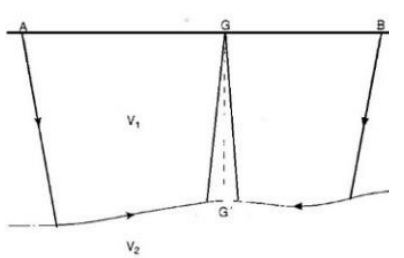

(a)

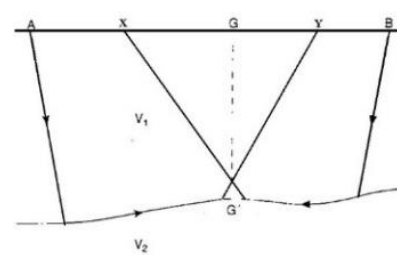

(b)
Figure 2. Representation of the minimum and maximum $X Y$ distances in a geophone $\mathrm{G}$.
These values are discrete, since they also depend on the distances between the geophones. Velocity functions are obtained for all $X Y$ values (Eq. 1).

After obtaining all the velocity functions for each of possible values for $X Y$, first-degree polynomial approximations are made to each function, to determine the softest curve. Coefficients for a polynomial $p(x)$ of degree 1 (in the sense of least squares) are found for data of the velocity function.

Then, we evaluate data we have from the position of geophones in the polynomial found to determine which of these velocity functions have lower quadratic error with respect to functions found and data obtained from the velocity function, with this, we determine the optimal $X Y$ value.

\section{Robust optimal $X Y$ value}

The process of finding the optimal $X Y$ value described above, is performed for each set of curves that is obtained by introducing noise in the original data. With this, we have a set of optimal $X Y$ curves of which the most representative curve must be found, in this case the median curve described by Fraiman \& Muniz (2001) is used.

\section{Median curve}

Using the theory of functional data, we seek to find the median curve or functional media. With this process, we want to find the curve that remains for the longest time in the center of the curve set. For this, the depth definition described by Fraiman \& Muniz (2001) is used. Obtaining the median curve corresponding to one of the velocity functions with optimal $\boldsymbol{X Y}$ values, we obtain the optimal robust $\boldsymbol{X} \boldsymbol{Y}$ value, which is used to find the timedepth function with velocity of the medium, using Eq. 2 and the depth in each geophone with Eq. 3 and in this way obtain how the subsoil is constituted.

\section{Numerical examples}

\section{Horizontal layers}

A first numerical experiment is carried out with a two horizontal layer model to obtain the arrival times using the equation

$$
t=t_{1}+\frac{x}{V_{2}}
$$

where

$$
t_{1}=\frac{2 h\left(V_{2}^{2}-V_{1}^{2}\right)^{1 / 2}}{V_{1} V_{2}}
$$

and to graph the direct and inverse times considering the critical point $x_{c}$ which is calculated at through the equation

$$
x_{c}=2 h \sqrt{\frac{V_{2}-V_{1}}{V_{2}+V_{1}}}
$$

The velocity of the first medium is $1400 \mathrm{~m} / \mathrm{s}$ and the velocity of the second medium is $4500 \mathrm{~m} / \mathrm{s}$; The horizontal distance is $69 \mathrm{~m}$ and the separation between the geophones is $3 \mathrm{~m}$; The total number of geophones is 24 on the surface. 
Several repetitions $(N)$ are made introducing uniform noise $(\zeta)$ in the position of the geophones to obtain different timedistance curves. With $N=100, N=500$, and $N=1000$, we use three different noises: $\zeta \sim U, \zeta \sim N(0,1)$, and a red noise.

Figure 3 shows the data with a uniform noise, with normal noise, and with a red noise for $N=100$.

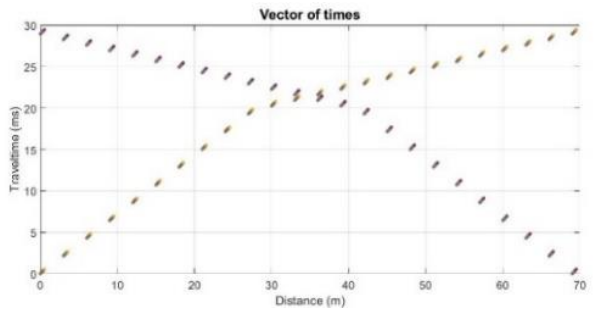

(a)

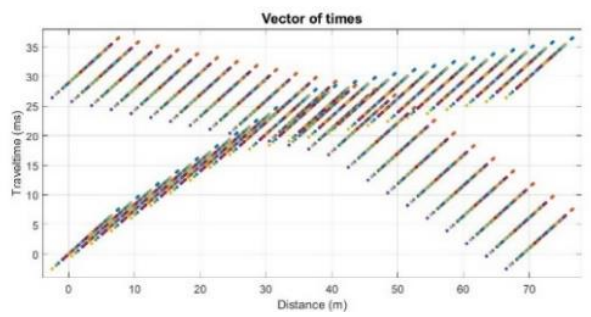

(b)

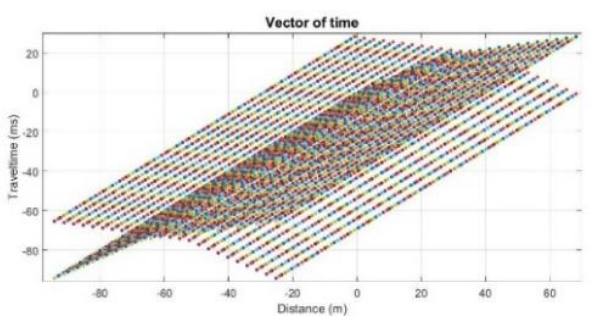

(c)

Figure 3. Distance-Time curves with variation in arrival times with $N=100$, and (a) $\zeta \sim U$, (b) $\zeta \sim N(0,1)$, and (c) a red noise

Figure 4 shows the data with a uniform noise, with normal noise, and with a red noise for $N=500$.

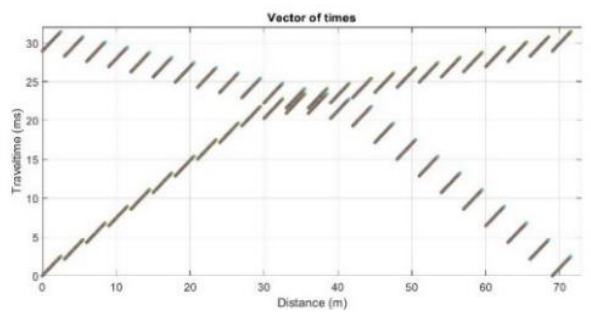

(a)

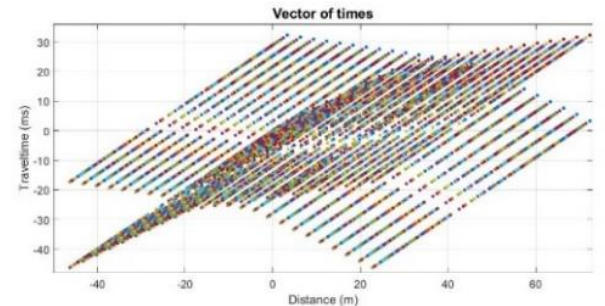

(b)

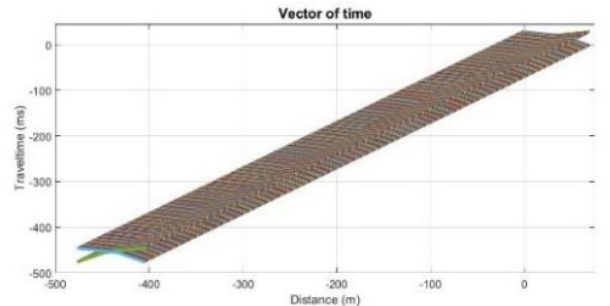

(c)

Figure 4. Distance-Time curves with variation in arrival times with $N=500$, and (a) $\zeta \sim U$, (b) $\zeta \sim N(0,1)$, and (c) a red noise.

Figure 5 shows the data with a uniform noise, with normal noise, and with a red noise for $N=1000$.

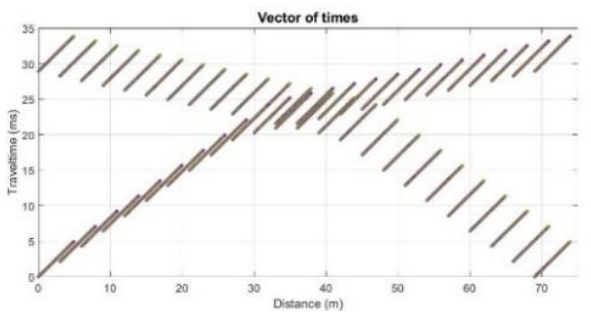

(a)

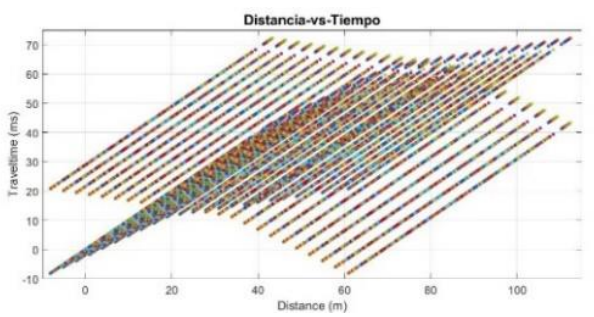

(b)

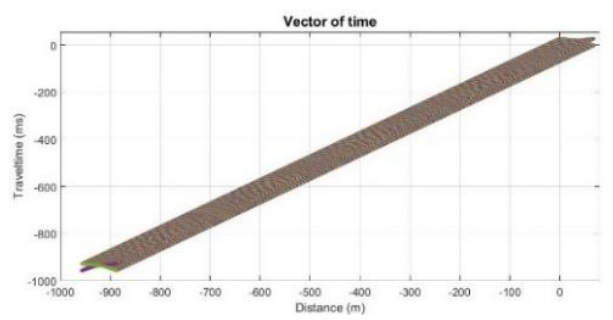

(c)

Figure 5. Distance-Time curves with variation in arrival times with $N=1000$, and (a) $\zeta \sim U$, (b) $\zeta \sim N(0,1)$, and (c) a red noise. 
After this, the velocity analysis function is Distance-Time curves with variation in arrival times with $\mathrm{N}=1000$, and (a) $\zeta \sim U$, (b) $\zeta \sim N(0,1)$, and (c) a red noise calculated for each of time vector data groups and its respective optimal $X Y$ value, thus generating an optimum velocity analysis function curve and then determine the median curve. Within each group, regardless of the type of noise incorporated, the solution is the same.

Figure 6 shows results of the median curve and the robust optimal $X Y$ for each of the $\mathrm{N}$ values.

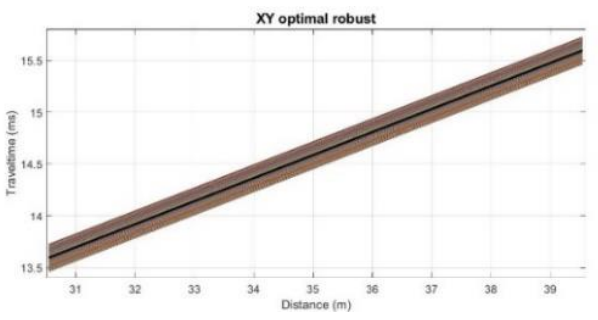

(a)

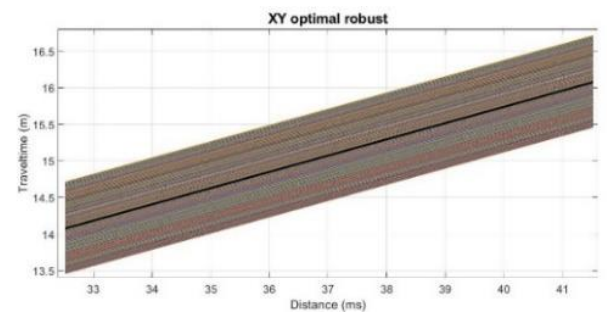

(b)

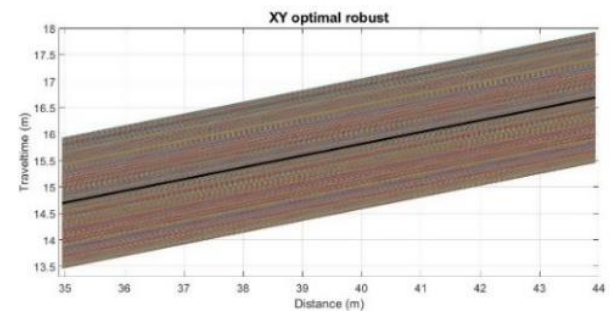

(c)

Figure 6. Median of optimal $X Y$ curves (a) $N=100$, (b) $N=500$, and (c) $N=1000$.

Thus, the optimum value of $X Y$ is $30 \mathrm{~m}$ and the velocity of the second medium is $4500 \mathrm{~m} / \mathrm{s}$ as expected.

\section{Irregular layer model}

This model was taken from Palmer (2001) and it has a flat surface and a totally irregular refractor. Geophones are located on the surface and are separated every $5 \mathrm{~m}$ on the surface to cover $335 \mathrm{~m}$ in total as shown in Figure 7.

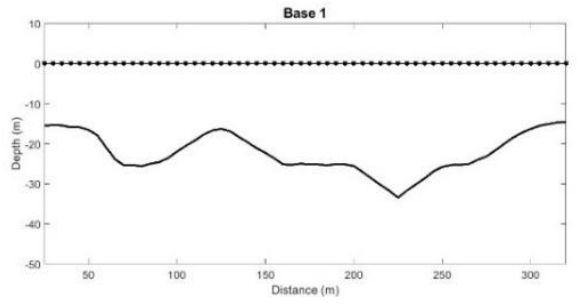

Figure 7. Simulated data with a flat surface and an irregular refractor

The same procedure used for the horizontal layer model is used. Arrival times are obtained by introducing a noise corresponding to $\zeta \sim U, \zeta \sim N(0,1)$, and a red noise, with $N=$ 500 (Figure 8) and $N=1000$ (Figure 9).

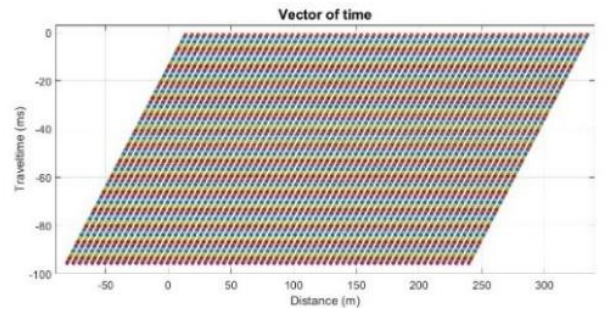

(a)

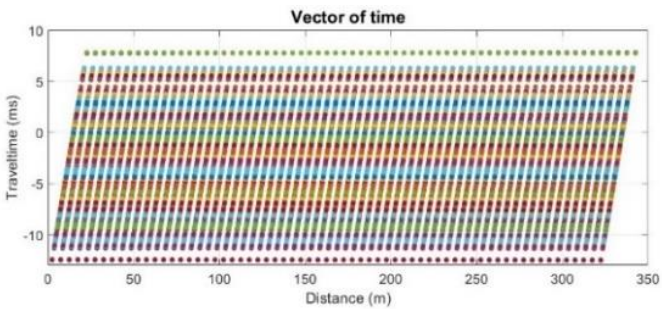

(b)

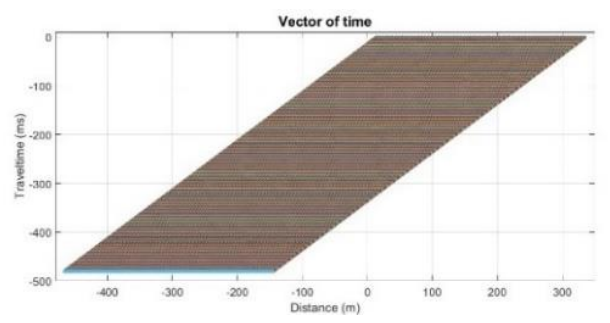

(c)

Figure 8. Distance-Time curves with variation in arrival times with $N=500$, and (a) $\zeta \sim U$, (b) $\zeta \sim N(0,1)$, and (c) a red noise.

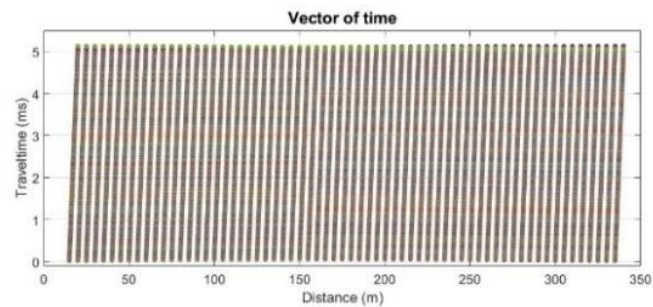

(a)

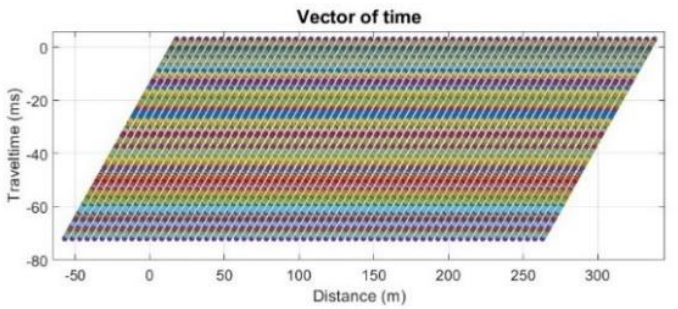

(b) 


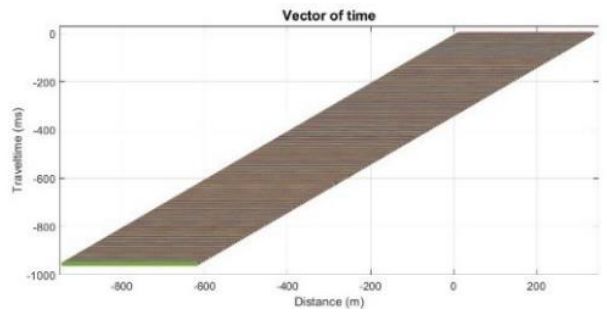

(c)

Figure 9. Distance-Time curves with variation in arrival times with $N=1000$, and (a) $\zeta \sim U$, (b) $\zeta \sim N(0,1)$, and (c) a red noise.

Figure 10 shows found medians curves, which in all cases we determine that the $X Y$ optimal robust is $20 \mathrm{~m}$ and the velocity of the refractor is $4421.518055 \mathrm{~m} / \mathrm{s}$.

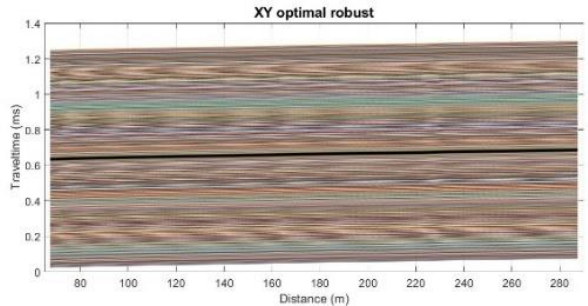

(a)

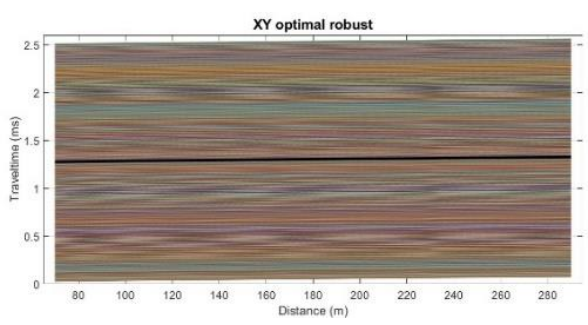

(b)

Figure 10. Median of optimal $X Y$ curves (a) $N=500$, (b) $N=1000$.

Figure 11 shows the found model compared to the original model shown in Figure 7.

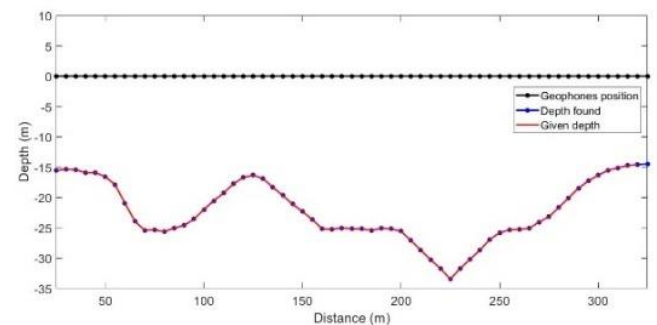

Figure 11. Depth model found for simulated data compared to the original model.

We can see that the found model has a good accuracy to the original model

\section{Conclusions}

The generalized reciprocal method is useful to define complex models with much more easily and accurately than most existing seismic refraction methods. This method depends on an appropriate determination of the optimal value of $X Y$, which at the optimum value, forward and reverse rays to each geophone emerge from near the same point on the refractor. GRM adds uncertainties in its application, using heuristic assumptions, without having mathematical basis.

We proposed an easy and understandable algorithm based on stochastic models and functional data analysis that allows to find a simple solution to the calculation of the optimal value $X Y$. The robust solution for parameter estimation in the GRM for seismic refraction allows to reduce the uncertainties associated with the calculation of the optimal value $X Y$.

Results were promising and resulting deterministic models are very close to original models demonstrating its high accuracy.

\section{Acknowledgments}

We would like to express our gratitude with Scientific Computing Apolo and the Department of Mathematical Sciences at Universidad EAFIT for supporting this work.

\section{References}

Chilusa, A. G., Naranjo, S. Exploración de subsuelo,121 pp, 2014

Fraiman, R., Muniz, G. Trimmed means for functional data. Test, 10(2), p. 419-440, 2001.

Leung, T.M. Examination of the optimum $X Y$ values by ray tracing. Geophysics, 60, p. 1151-1156, 1995.

Leung, T.M. Controls of travel time data an problems of the generalized reciprocal method. Geophysics, 68, p. 16261632, 2003.

Palmer, D. The generalized reciprocal method of seismic refraction interpretation, Society of Exploration Geophysicists, Tulsa, 113 pp, 1980

Palmer, D. An introduction to the generalized reciprocal method of seismic refraction interpretation. Geophysics, 46 (11), p. 1508-1518, 2001.

Sjorgen, B. A brief study of application of the generalized reciprocal method and of some limitations of the method. Geophysical prospecting, 48, p. 815-834, 2000.

Whiteley, R. J. and Eccleston, P. J. Comparison of shallow seismic refraction interpretation methods for regolith mapping. Exploration Geophysics, 37, p. 340-347, 2006. 